\title{
MORPHOLOGY AND OPTICAL PROPERTIES OF LASER-ASSISTED CHEMICAL VAPOUR DEPOSITED GaN
}

\author{
E.M. Goldys ${ }^{a}$, M. GodLeWski ${ }^{b}$ AND T.L. TANSLeY ${ }^{a}$ \\ ${ }^{a}$ Semicond. Sci. \& Technol. Lab., Macquarie Univ. \\ North Ryde, 2109 NSW, Australia \\ ${ }^{b}$ Institute of Physics, Polish Academy of Sciences \\ Al. Lotników 32/46, 02-668 Warsaw, Poland
}

\begin{abstract}
Properties of GaN epilayers grown by laser-assisted chemical vapour deposition are discussed. Good crystallinity and surface morphology of the films is demonstrated. Micro-Raman spectra are explained by scattering by small, randomly oriented cubic phase units present in the GaN film.
\end{abstract}

PACS numbers: 68.55.-a, 81.15.Gh, 61.16.Ch, 78.30.-j

Laser-assisted chemical vapour deposition (LCVD) has become an attractive technology in the electronic industry over the past decade. One of the key benefits of LCVD is that instead of using the thermal energy, LCVD takes advantage of photon energy to induce chemical reactions between reactants and deposit films. In this work we are using the photolytic process in which the ArF $193 \mathrm{~nm}$ excimer laser beam is absorbed by the precursor gases. The deposition of $\mathrm{GaN}$ was carried out at temperatures around $600^{\circ} \mathrm{C}$. Low growth temperatures, between $470^{\circ} \mathrm{C}$ and $680^{\circ} \mathrm{C}$, make possible the use of a range of thermally unstable substrates, such as silicon, gallium arsenide, quartz glass, in addition to sapphire and $\mathrm{SiC}$.

GaN films grown by LCVD with a range of ammonia flow rates show a systematic variation in electrical parameters. The Hall mobility increases proportionally with flow rate, from $55 \mathrm{~cm}^{2} /(\mathrm{V} \mathrm{s})$ to $90 \mathrm{~cm}^{2} /(\mathrm{V} \mathrm{s})$, saturating at $95 \mathrm{~cm}^{2} /(\mathrm{V} \mathrm{s})$ at flow rates above about $100 \mathrm{ml} / \mathrm{min}$. The electron carrier concentration decreases monotonically and reproducibly from $10^{17} \mathrm{~cm}^{-3}$ to $6 \times 10^{14} \mathrm{~cm}^{-3}$ over the flow rate range 0 to $100 \mathrm{ml} / \mathrm{min}$. The sharp increase in the Hall mobility indicates that the electron scattering inside the film has been reduced. Nitrogen vacancies are therefore assumed to be reduced, as growth conditions are not changed other than by the addition of excess nitrogen via the ammonia plasma. Electron concentration continues to fall beyond mobility saturation, suggesting that the reduction in nitrogen vacancy concentration thought to be responsible for large $n$-type autodoping, is accompanied by compensation, possibly by increasing the density of other defects caused by ion damage. 
Despite low growth temperature the LCVD-grown films show cathodoluminescence spectra similar in character to those observed in MBE and MOCVD grown films. The band-gap emission is observed as well as the yellow band. Cathodoluminescence images taken at near-band-gap wavelengths and within the yellow band show a high degree of uniformity, in contrast to MOCVD and MBE-grown $\mathrm{GaN}$ where granular structures is clearly visible in the band-gap emission. Raman spectra of the LCVD GaN films grown in a range of substrate temperatures both on sapphire and on quartz are shown in Fig. 1. The spectra were taken using a Renishaw micro-Raman system and an Ar laser excitation. The main feature is a broad peak with its maximum at $725-733 \mathrm{~cm}^{-1}$ and of width approximately $40-80 \mathrm{~cm}^{-1}$, superimposed on a broader band with its maximum at about $580-610 \mathrm{~cm}^{-1}$. The presented spectra have been found to be independent of the in-plane orientation of the substrate, of the laser polarization and of the polarization of the scattered light. The spectra are much broader than usually reported for high quality GaN films [1-3] but they strongly resemble those obtained from the films with mixed crystalline and amorphous phases observed in GaAs, microcrystalline $\mathrm{Si}$ [4-6]. The presence of the amorphous component is typically associated with the broad $\mathrm{Ra}$ man feature at an energy lower than that of the dominant Raman active mode in

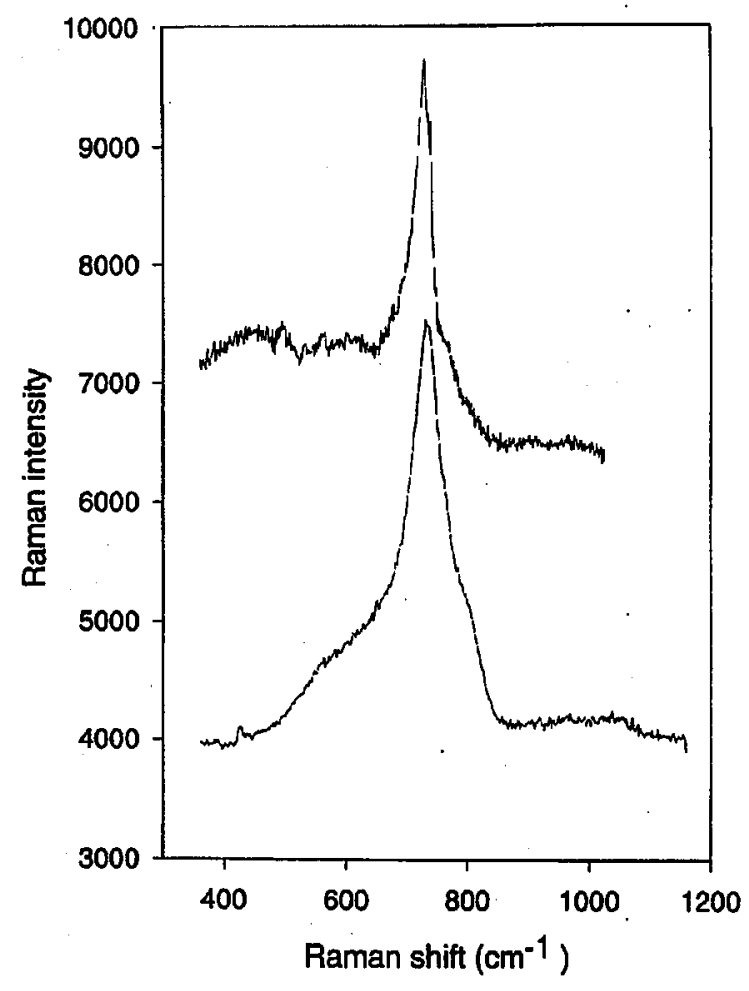

Fig. 1. Raman spectra of LCVD GaN films grown on quartz (top) and on sapphire (bottom). 
the crystalline phase [4, 5]. Tabata et al. [1] have also observed a similar broad line shape in apparently wurtzite GaN. In hexagonal GaN the dominant Raman peak occurs at around $560 \mathrm{~cm}^{-1}$, while the lines around $740 \mathrm{~cm}^{-1}$ are weaker. The $740 \mathrm{~cm}^{-1}$ has been observed in more recent works where, we believe, a cubic phase may have been present $[1,7]$, and also in films with a low electron concentration. On the other hand, in cubic GaN the intense LO peak at about $740 \mathrm{~cm}^{-1}$ dominates and the forbidden TO peak at about $560 \mathrm{~cm}^{-1}$ is much weaker [1]. The dominant peak in Fig. 1 should correspond to the most intense Raman active mode in GaN $[1,2,8]$. Our Raman spectra are thus attributed to scattering by small, randomly oriented cubic phase units present in the film.

The broadening of the Raman line is now discussed. The observed large Raman linewidth compared with the width of Raman lines in crystalline GaN is interpreted using the concept of finite coherence length of phonons in the examined films. The presence of crystallites results in the spectral characteristics of Raman intensity modelled by the following expression [9]:

$$
I(\omega)=\int_{0}^{\infty} \frac{\mathrm{d}^{3} q q^{2} \exp \left(-l^{2} q^{2} / 4\right)}{[\omega-\omega(q)]^{2}+\Gamma^{2}},
$$

where $l$ is the phonon coherence length, related to the presence of crystallites, $q$ is the phonon wave vector, $\Gamma$ is the Raman width in the absence of crystallites, $\omega(q)$ is the phonon dispersion relationship. The key effect of finite phonon coherence length is the relaxation of the wave vector selection rules, thus allowing phonons with non-zero wave vectors to take part in Raman scattering, causing the increased width of the Raman peak. In this approach, vibrational modes of small crystallites are described as phonons with an uncertainty in the wave vector $\Delta k \approx 2 \pi / d$, where $d$ is the crystallite size, and the momentum selection rules for the Raman process are therefore relaxed. The increased width of the modelled Raman intensity is accompanied by asymmetry, the peak intensity also shifts to lower energies (referred to as "softening" of phonon modes). By using the crystallite size. of $6.5-7 \mathrm{~nm}$ we have obtained the peak red shifts down to $728-731 \mathrm{~cm}^{-1}$ and the linewidth of $40 \mathrm{~cm}^{-1}$, close to the observed value. The value of $7 \mathrm{~nm}$ should be used as an indication of a typical crystallite size only, in a more realistic model a distribution of the crystallite sizes' should be used.

Small-scale crystallites or grams typically around $10 \mathrm{~nm}$ are clearly visible in high-resolution intages taken by the field emission microscope (JEOL JSM-6300F) (Fig. 2a) of the surface of LCVD-grown films. This feature size is in agreement with earlier observations of Fuji et al. [10]. The crystallites are uniform in size and are uniformly distributed on the sample surface. As a result the surface topography is very smooth. Such smooth surface morphology is in distinct contrast with surface morphologies of $\mathrm{GaN}$ grown by MBE in both wurtzite and cubic phases, as well as those of the MOCWVD-grown GaN (Fig. 2b-d). In MBE-grown GaN distinctive hillocks are often visible - these can have hexagonal base in wurtzite $\mathrm{GaN}$ as well as a square base in cubic GaN. MOCVD GaN, which is grown at much higher temperatures, exhibits very uneven and irregular surface morphology, likely to be related to high surface mobilities of the adatoms during growth. It is underlined, however, that in addition to temperature, high growth pressure must be a deci- 

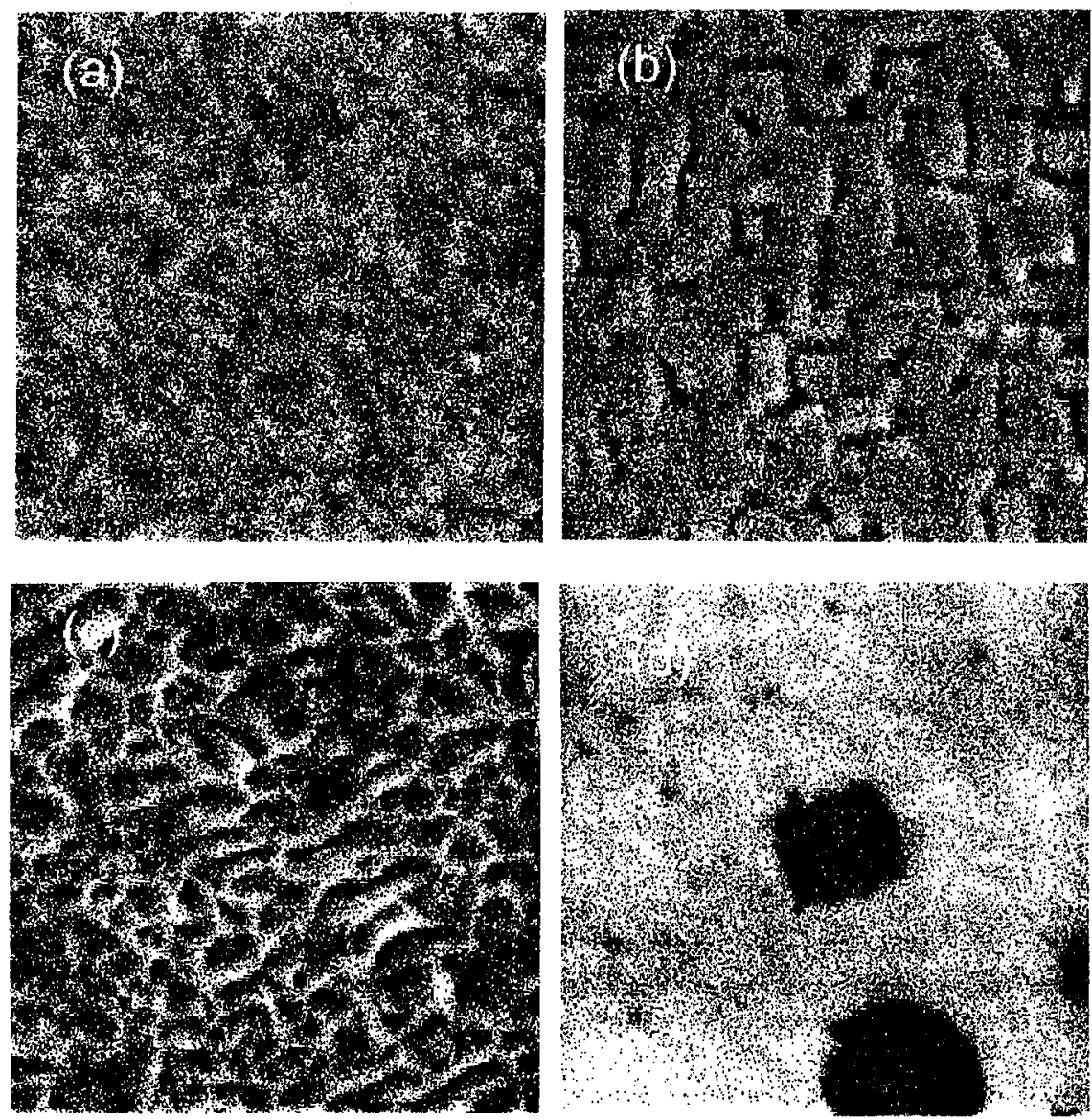

Fig. 2. Field emission images of the surface of the (a) LCVD-GaN film $(1.7 \times 1.7 \mu \mathrm{m})$, (b) cubic MBE-grown GaN $(5 \times 5 \mu \mathrm{m})$, (c) hexagonal MBE-grown GaN $(5 \times 5 \mu \mathrm{m})$, (d) MOCVD-grown GaN $(5 \times 5 \mu \mathrm{m})$.

sive factor in determining the surface morphology of the LCVD GaN. We note that surface morphology of LCVD GaN and that of MBE GaN both grown on Si are very different, the LCVD being superior. This characteristics of LCVD films is particularly beneficial for these microelectronics device applications for which LCVD growth is appropriate.

\section{References}

[1] A. Tabata, R. Enderlein, J.R. Leite, S.W. da Silva, J.C. Galzerani, D. Schikora, M. Kloidt, K. Lischka, J. Appl. Phys. 79, 4137 (1996).

[2] H.R. Kuo, M.S. Feng, J.D. Guo, M.C. Lee, Jpn. J. Appl. Phys. 34, 5628 (1995).

[3] S. Murugkar, R. Merlin, A. Botchkarev, A. Salvador, H. Morkoc, J. Appl. Phys. 77, 6042 (1995). 
[4] I.D. Desnica, M. Ivanda, M. Kranjcec, R. Murri, N. Pinto, J. Non-Cryst. Solids 170, 263 (1994).

[5] R. Zallen, J. Non-Cryst. Solids 141, 227 (1992).

[6] R.J. Nemanich, E.C. Buehler, Y.M. Legrice, R.E. Shroder, G.N. Parsons, C. Wang, G. Lucovsky, J.B. Boyce, J. Non-Cryst. Solids 114, 813 (1989).

[7] M. Giehler, M. Ramsteiger, O. Brandt, H. Yang, K.H. Ploog, Appl. Phys. Lett. 67, 733 (1995).

[8] D.D. Manchon Jr, A.S. Barker, P.J. Dean, R.B. Zetterstrom, Solid State Commun. 8, $1227(1970)$.

[9] J. Yoshida, J. Appl. Phys. 67, 7283 (1990).

[10] H. Fuji, C. Kisielowski, J. Krueger, M.S.H. Leung, R. Klockenbrink, M. Rubin, E.R. Weber, in: Proc. III-V Nitrides Symposium, Boston 1996, Ed. F.A. Ponce, MRS, Pittsburgh 1997, p. 227. 